\title{
CONTRIBUCION AL CONOCIMIENTO DE LA PODREDUMBRE CASTAÑO-ROJIZA DE LA VAINA DEL ARROZ
}

A contribution to the knowledge of reddish-brown rot in the sheath of rice

M. A. Mazzanti de Castañón \& S.A. Gutiérrez de Arriola

Cátedra de Fitopatología,

Facultad de Ciencias Agrarias, U.N.N.E., Sargento Cabral 2131, 3400 Corrientes, Argentina

Palabras clave: Podredumbre castaño rojiza de la vaina, arroz, Helicoceras oryzae.

Key words: Reddish-brown sheath rot, rice, Helicoceras oryzae

\section{RESUMEN}

Se determinó la capacidad patogénica y la identidad de un hongo productor de esclerocios, asociado a lesiones de vainas foliares, detectado en cultivos de arroz regados por inundación en el nordeste de la Argentina. Nuestros objetivos fueron diferenciarlo de otros hongos patógenos semejantes en estos cultivos de la región y obtener información sobre la enfermedad denominada podredumbre castaño-rojiza de la vaina del arroz.

Se aplicaron métodos comunes para el aislamiento e identificación de hongos fitopatógenos. Se inocularon semillas in vitro y plantas cultivadas en macetas con riego por inundación.

Su forma conidial coincidió con el de Helicoceras oryzae Linder \& Tullis, agente causal de la podredumbre castaño-rojiza de la vaina de arroz, el cual se comportó como patógeno débil en las pruebas de patogenicidad.

Por su morfologia, $\boldsymbol{H}$. oryzae (Hyphomycetes Helicosporae) debiera incluirse en el género Dichotomophthoropsis M.B. Ellis.

\section{INTRODUCCION}

Los antecedentes encontrados sobre Helicoceras oryzae son escasos. En Japón se lo cita como agente causal de la podredumbre castaño-rojiza de la vaina (reddish-brown sheath rot), una de las enfermedades escleróticas del arroz $(8,11)$. Además, se lo asocia con la descoloración o manchado de granos de arroz, figurando entrelos hongos de campo que son más o menos parasiticos

\section{SUMMARY}

The pathogenical capacity as well as the identity of a fungus forming sclerotia, associated with lesions in leaf sheaths, and which was detected in rice cultivations watered through flooding in northeast Argentina was determined. Our objectives were to differentiate the former from other pathogenical fungi which are similar in these cultivations of the region and to get some informations about the so called reddish-brown root disease in the sheath of rice.

In order to isolatedandidentify phytopathogenic fungi, current methods were used. Seeds in vitro and plants cultivated in flower-pots through flooding watering were inoculated. Their conidial form coincided with that of Helicoceras oryzae Linder \& Tullis, the causing agent of the reddish-brown rot in the sheath of rice, which behaved like a weak pathogenus in the pathogenical tests.

Due to its morphology, H. oryzae (Hyphomycetes, helicosporae) should be included in the genus Dichotomophthoropsis M.B.Ellis.

y colonizan la semilla antes de la cosecha $(1,8,9)$.

De acuerdo a Ou (8), Helicoceras Linder 1931, se considera como sinónimo de Sirosporium Bubák \& Serebrianikow 1912. A este género más antiguo que tiene prioridad, ha sido transferida una de las especies del primero (3), sin embargo $\boldsymbol{H}$. oryzae, se mantiene en la actualidad dentro del Helicoceras.

Goos (3) consigna que $\boldsymbol{H}$. oryzae es un nomen confusum, mientras Lee \& Gunnell (5), lo incluyen en una lista de hongos señalados como agentes de enfermedades atipicas o raras del follaje y glumas de arroz, donde la infección puede apreciarse cuando las

- Trabajo financiado por la Secretaria General de Ciencia y Téenica de la Universidad Nacional del Nordeste, U.N.N.E., Corrientes, Argentina. 
plantas estaban debilitadas, moribundas, o bajo condiciones ambientales adversas, siendo probable que algunos de estos hongos, sean patógenos débiles o saprófitos.

Durante la estación de cultivo 1993-1994, en reconocimientos de arrozales de las provincias de $\mathrm{Co}$ rrientes y de Formosa, se recolectaron vainas foliares que presentaban áreas descoloridas, con numerosos puntos oscuros visibles a simple vista. En el laboratorio se comprobó que tales puntos eran esclerocios, de aspecto diferente a otros hongos patógenos del arroz en el nordeste de la Argentina (NEA).

La siembra del material enfermo permitió el desarrollo de un hongo con conidios helicoidales semejante al Hyphomycete Helicoceras oryzae Linder \& Tullis $(6,8,9)$, una especie hallada en Texas, USA, sobre granos de arroz chino (6). Posteriormente, en las colonias se observó la formación de esclerocios similares a los observados en las vainas foliares.

En vista de la importancia del cultivo para la región y ante la presencia de un hongo no citado en el país, nuestros objetivos fueron: determinar la capacidad patogénica y la identidad del hongo, asociado a lesiones en vainas foliares, a fin de diferenciarlo de otros hongos patógenos semejantes de estos cultivos en la región.

\section{MATERIALES Y METODOS}

Se estudiaron muestras de plantas de arroz enfermas de los cvs. BR-IRGA 409 y Fortuna, y de dos líneas en mejoramiento, que se obtuvieron de cultivos particulares y ensayos de la Estación Experimental Agropecuaria Corrientes, I.N.T.A., regados por inundación, en los Dptos. Berón de Astrada, Goya, Santo Tomé (Corrientes) y Laishi (Formosa). Los trabajos de laboratorio se realizaron en la Facultad de Ciencias Agrarias, U.N.N.E.

SINTOMATOLOGIA. Las muestras fueron examinadas a simple vista y con microscopio estereoscópico (60x). Para apreciar la evolución del material enfermo, se colocaron trozos de vainas dentro de cámaras húmedas, en condiciones de laboratorio y en estufa regulada a $25^{\circ} \mathrm{C}$.

SIEMBRAS. Se efectuaron siembras comunes de:

1) Esclerocios formados en el substrato natural

2) Trocitos de vainas foliares tomados del límite entre el tejido sano y enfermo. El material se desinfectó con hipoclorito de sodio al $2,5 \%$ durante un minuto y se sembró en: agar agua (AA) al $2 \%$, agar papa dextrosa (PDA) al 1,5\%, pH 7. y agar papa zanahoria (PCA).

A partir de esclerocios y de conidios desarrollados en las siembras comunes, se realizaron aislamientos de puntas de hifas y cultivos monospóricos respecti- vamente PDAy PCA.

Se examinaron las las características morfométricas del hongo $(600 \mathrm{x})$ en el substrato natural y en los aislamientos comunes, desde puntas de hifas y los cultivos monospóricos; . Se observaron las características culturales de las colonias desarrolladas durante 15 días en condiciones de laboratorio, $12 \mathrm{~h}$ luz-12 $\mathrm{h}$ oscuridad. a $25-27^{\circ} \mathrm{C}$.

Los aislamientos estudiados se guardaron en la Cátedra de Fitopatología de la Facultad de Ciencias Agrarias, U.N.N.E.

PRUEBAS DE PATOGENICIDAD. Se inocularon semillas de arroz in vitro y plantas de 43 y de 70 días (estados vegetativo y reproductivo, respectivamente), criadas en macetas al aire libre y en piletas con riego por inundación.

Se probó la patogenicidad de dos aislamientos del hongo obtenidos de material procedente de Formosa, uno monospórico (sobre semillas) y otro de punta de hifa (sobre plantas), que desarrollaron durante 16 días en $\mathrm{PDA}$ en placas de Petri, a $19-26^{\circ} \mathrm{C}$ (prueba con semillas) y a $27-31^{\circ} \mathrm{C}$ (pruebas con plantas).

En todos los casos se realizaron reaislamientos a partir del material inoculado.

A. Inoculación de semillas. Semillas sanas de los crs. BR-IRGA 409 y CT 6919 se desinfectaron con hipoclorito de sodio $2,5 \%$ durante 10 minutos.

Con sacabocados de $3 \mathrm{~mm}$ de diámetro se cortaron discos de inóculo, que se llevaron a cámaras húmedas en placas de Petri en condiciones de esterilidad; sobre cada disco se colocó una semilla ( 20 por cada cv.). Los testigos fueron semillas con y sin desinfectar, colocadas en placas de Petri preparadas de la misma forma, sobre discos de PDA estéril.

Las placas se mantuvieron en condiciones de laboratorio, 12 horas luz-12 horas oscuridad; a los seis días se determinó el poder germinativo, que en ambos cultivares fue del $90 \%$. Las observaciones se realizaron durante 11 días, lapso en que la temperatura varió entre $20-27^{\circ} \mathrm{C}$.

B. Inoculación de plantas. Dos días antes de la inoculación, las macetas con plantas sanas del cv. BR-IRGA 409 de 43 días, fueron llevadas al laboratorio y se cubrieron con bolsas de polietileno.

Se inocularon en total 38 macollos. Con ayuda de agujas metálicas estériles se introdujeron discos de inóculo de $3 \mathrm{~mm}$ de diámetro, entre la vaina foliar inferior y el tallo; luego, en 25 de las plantas tratadas se produjeron heridas (tres pinchazos con aguja estéril) atravesando la vaina, en el lugar donde se depositó el inóculo. En todos los casos se cubrió con cinta adhesiva transparente. 
Se utilizaron plantas testigos en las que se colocaron discos de PDA estéril, las cuales se trataron en la misma forma, 10 sin heridas y 8 con heridas. Luego de los tratamientos, las plantas se cubrieron con bolsas de polietileno que se quitaron a las 24 horas y las macetas se retornaron a las piletas, separando las testigos de las inoculadas.

La prueba de patogenicidad con plantas del cv. BR-IRGA 409 de 70 días, se realizó de acuerdo a lo detallado; se inocularon 8 plantas con heridas y 8 sin heridas y se llevaron 10 y 7 testigos, con y sin heridas, respectivamente.

Durante los 30 días posteriores a la inoculación, las temperaturas registradas en el exterior variaron entre 18,5 y $40^{\circ} \mathrm{C}$.

\section{RESULTADOS}

Las lesiones se observaron con características semejantes, en plantas en el estado de maduración, en la campaña 1993-1994 en el cv. BR-IRGA 409 (arroz semienano) y en dos líneas en mejoramiento, Dptos. Santo Tomé (Corrientes) y Laishi (Formosa); y en la campaña 1994-1995 en el cv. Fortuna (arroz de porte alto), Dptos. Berón de Astrada y Goya (Corrientes).

SINTOMATOLOGIA. En las plantas del campo, en vainas foliares inferiores bajo la línea de agua se observaron áreas descoloridas, irregulares, en las que se distinguen a simple vista numerosos puntos oscuros. Tales puntos son esclerocios $(60 \mathrm{x})$, formados en el interior de la vaina; al romperse la epidermis, se aprecia un aspecto carbonoso e irregular (Fig. 1).

En vainas de plantas inoculadas artificialmente (Fig. 2), aparecen lesiones alargadas, irregulares, de tamaño variabie, al principio de aspecto húmedo, oscuras, luego de color castaño rojizo oscuro que cuando envejecieron se tornaron descoloridas, apagadas, perdurando el matiz rojizo.

Con el tiempo las vainas se secaron y en su interior se formaron los esclerocios del hongo.

ETIOLOGIA. En los aislamientos comunes, de puntas de hifas y monospóricos en PCA, a los cinco dias de la siembra se observó la formación de abundantes conidios muy grandes de color canela, hundidos y en la superficie del medio, en el centro y en los bordes de la colonia. Un día después los mismos conidios se observaron en PDA y en AA. A los siete dias, en PDA y PCA se encontraron esclerocios semejantes a los formados en el interior de la vaina foliar; dichos cuerpos no desarrollaron en AA.

En la fase conidial del hongo, los aislamientos en AA, PDA y PCA asemejaban al del Hyphomycete descripto bajo el nombre Helicoceras oryzae Linder \& Tullis $(6,8,9)$. En PDA, a los 15 días de la siembra el microorganismo presentaba las características morfométricas siguientes:

Hifas tabicadas, algo coloreadas, diámetro regular, paredes lisas; conidióforos (Fig. 3) diferenciados de las hifas vegetativas, ramificados, tabicados, color castaño claro, paredes lisas, terminando en células conidiógenas lobadas politréticas con poros visibles en la pared exterior; conidios (Figs. 4, 5) secos, muy grandes, de (92,5-) 117,5 (-132,5) x (8,7-) 9,8 (-10) $\mu \mathrm{m}$, solitarios en cada lóbulo, cilíndricos, curvos, uncinados a circinados, enrollados en 1-3 espirales aproximadamente, equinulados, de extremos redondeados, color castaño claro, con 7-14 ó más tabiques, estrechadosen los septos, células por lo general un poco más largas que anchas; esclerocios (Figs. 1, 6) polimórficos, globosos, subglobosos o irregulares, de color oscuro, fuscos, opacos, diámetro (135-) $245,6(-325) \times(100-) 194,5(-250) \mu \mathrm{m}$, células superficiales pequeñas, irregularmente poligonales.

Las medidas de los conidios corresponden mayoritariamente a los más derechosy deformacilíndrica.

Los esclerocios obtenidos del substrato natural (vainas de arroz) midieron (175-) 237,8 (-360) x (150-) $208,9(-315) \mu \mathrm{m}$.

De acuerdo a los términos propuestos por Goos (3), , los conidios del hongo del NEA son semicircinados circinados y tortuosos (ya sea con un enrollamiento parcial, o desde 1 a 5 espirales)(fig. 4-5).

En PDA, la colonia es de crecimiento radial regular, cubre el fondo de la placa, con micelio aéreo de color castaño oscuro, verdoso, con abundantes conidios y esclerocios aislados o agrupados, hundidos y en la superficie del medio, reverso de aspecto semejante, con tonos más oscuros.

PRUEBAS DE PATOGENICIDAD. Todas las pruebas de patogenicidad dieron resultados positivos, y el hongo inoculado fue reaislado de los tejidos enfermos.

A. Inoculación de semillas. Los dos cvs. manifestaron los primeros sintomas a los 7-8 dias de la inoculación. Se observaron plántulas con radículas cortas, oscurecidas, 0 sin radículas, coleóptilos de escaso desarrollo, ligeramente oscurecidos y semillas sin germinar, cubiertas por micelio castaño verdoso; sobre las semillas se formaron los conidios del hongo inoculado.

A los diez dias se detectaron los esclerocios que también se formaron sobre las semillas, y en radículas y ápices de plántulas muertas. Al finalizar la prueba, la totalidad de las plántulas estaban muertas, con abundante micelio y esclerocios sobre las mismas. Las plantas 
testigos no presentaron anormalidades.

B. Inoculación de plantas. Las primeras reacciones aparecieron al cuarto día de la inoculación en las plantas de 70 días y un día después en las de 43 días. Se observó oscurecimiento de la vaina foliar en el lugar donde se depositóel inóculo, el cualfue más notorioen el tratamiento con heridas. Veinticuatro horas más tarde el oscurecimiento era igualmente perceptible en todas las plantas inoculadas, sin y con heridas.

La sintomatología evolucionó de acuerdo a loya descrito. Durante las observaciones las plantas testigos no manifestaron síntomas.

\section{DISCUSION}

Linder (6), incluyó cuatro especies en el género Helicoceras: H. celtidis, H. plantaginis, H. nymphearumy $H$. oryzae. Según Goss (3), tres han sido transferidas: H. celtidis a Sirosporium, H. nymphearum a Dichotomophthoropsis, y H. plantaginis a 4 iolella. En el género Helicoceras se han descripto dos especies más, H. longisporum Subram. y H. indicum P.R. Rao \& Dev Rao, por lo que el género mantiene 3 especies .

El hongo en la fase esclerotial, asociado con lesiones en vainas foliares de arroz, en varias localidades del NEA, produjo su fase conidial con características semejantes a las del Hyphomycete conocido bajo el nombre de Helicoceras oryzae Linder y Tullis $(6,8,9)$.

En reiteradas siembras yaislamientos a partir de esclerocios comunes y de puntas de hifas, se comprobó que la especie produce su fase conidial además de los esclerocios, en PDA y en PCA; en los aislamientos monospóricos, también se obtuvieron ambas formas juntas en los dos medios. En AA, desarrolló el estado conídico pero no se formaron esclerocios.

La bibliografía consultada no aporta mayores datos sobre la fase esclerotial del hongo, el que a primera vista podría confundirse con Sclerotium oryzae Catt., agente causal de la podredumbre del tallo de arroz, común en la región (7), que en condiciones de campo también se observó como puntos oscuros en vainas foliares inferiores, bajo de la linea de agua. Sin embargo, en esta enfermedad las lesiones fueron de color negro o muy oscuras y los esclerocios fueron producidos externamente sobre la cara interna de la vaina, pero principalmente el interior del tallo huecode arroz, cuando la podredumbre profundiza hasta dicho órgano.

$\mathrm{El}$ anamorfo $S$. oryzae, Nakataea sigmoidea (Cav.) Hara, se produce en AA y en PCA, pero no en PDA. De las dos variedades escleróticas que se conocen de dicho patógeno (var. sigmoidea, var. irregulare) (8), en el NEA se encuentra presente la primera, cuyos esclerocios globosos, regulares, lisos, negros, brillantes, se diferencian claramente de los de $\boldsymbol{H}$. oryzae. Se considera que los esclerocios de la var. irregulare, hasta la fecha no detectada en la región, por su morfología irregular podrían confundirse con los del hongo que motivó el presente trabajo.

Otro patógeno del arroz común en el NEA, Rhizoctonia oryzae Ryker y Gooch (4), agente causal de la mancha de la vaina (sheath spot), produce lesiones definidas, elipticas, con centros de color gris ceniciento y bordes bien desarrollados castaño oscuro. Sobre las manchas ocasionalmente se observan esclerocios en material procedente del campo, los que se producen en abundancia en PDA; tales esclerocios irregulares, de color anaranjado-salmonado, se diferencian con facilidad de los de H. oryzae (160 x).

Las especies de Sirosporium, presentan conidios que no se ajustan a las características de estos Hyphomycetes $(2,3)$; además, no se encontraron antecedentes de que formenesclerocios. Dichotomophthoropsis, presenta helicoconidios y puede producir además esclerocios $(2,3)$.

Sobre la base de los resultados obtenidos y de los pocos antecedentes bibliográficos encontrados, las opinamos que esta especie no corresponde al género Sirosporium (Figs. 1, 3, 4, 5, 6), pero es muy semejante al género Dichotomophthoropsis M.B. Ellis $(2,3)$. Esto se apoyaría en que a este género fue transferida una de las especies de Helicoceras como Dichotomophthoropsis nymphaearum (Rand) M.B. Ellis, = Helicoceras nymphaearum (Rand) Linder, la que causa manchas sobre hojas de Nymphaea spp. (2,3) (plantas acuáticas perennes que se hallan presentes en arrozales de la región).

Por otra parte, según Linder (6) H. nymphaearum y $\boldsymbol{H}$. oryzae se asemejan, ambas especies producen esclerocios, diferenciándose principalmente por el color y el tamaño de los conidios. En la India se denunció la transmisión por semilla de $\boldsymbol{H}$. nymphaearum, que ocasiona pudrición de la vaina y descoloramiento de granos de arroz (10). Por consecuencia, no se descarta que $\boldsymbol{H}$. oryzae y $H$. nymphaearum podrían ser la misma especie. La correcta nomenclatura y taxonomía del hongo deberán ser corregidas.

En las condiciones en que se realizaron las inoculaciones, $\boldsymbol{H}$. oryzae, demostró capacidad patógena sobre plántulas y plantas en estado vegetativo y en los inicios del estado reproductivo. No obstante, los efectos producidos por el hongo parecen no afectar mayormente la posterior evolución de las plantas, aunque las vainas inoculadas se secaron prematuramente. Por la demora en aparecer las primeros sintomas, parecería que se trata de un patógeno débil que actuaría sobre plantas que han sufrido algún tipo de estrés, en el caso presente, plántulas creciendo in vitro sin sustentación y plantas 

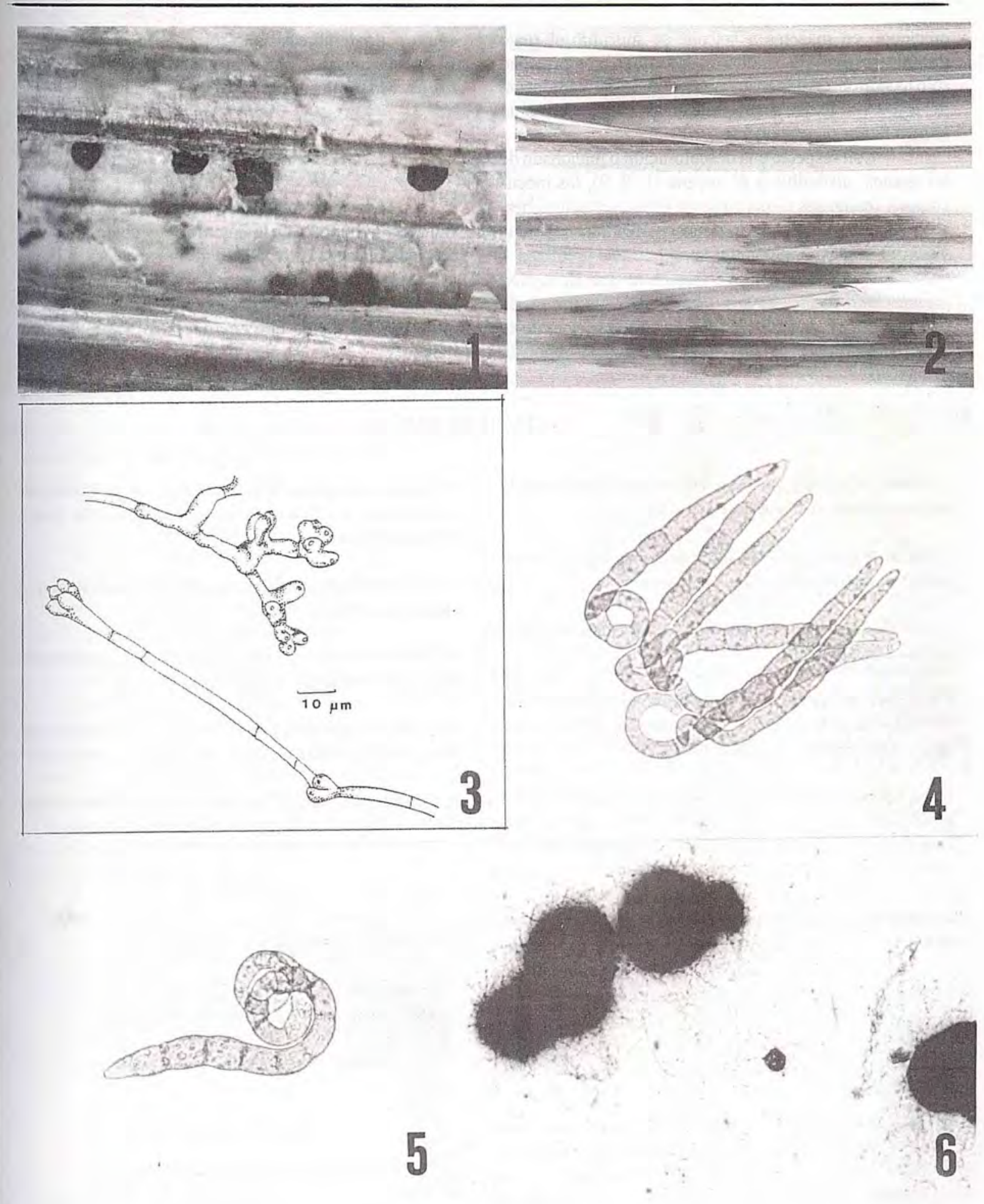

Fig. 1, 2, 3, 4, 5, 6. Helicoceras oryzae. Esclerocios formados en el interior de la vaina foliar del arroz, (60x). Fig. 2. Síntomas en vainas foliares de arroz cv. BR-IRGA 409, inoculadas artificialmente con $\boldsymbol{H}$. oryzae (abajo) y testigos correspondientes (arriba). Fig. 3. Dibujo de conidióforos en cámara clara. Fig. 4. Conidios semicircinados y circinados (400x). Fig. 5. Conidio con su ápice helicoidal (400x). Fig. 6. Esclerocios, desde un cultivo in vitro (200x). 
creciendo en macetas a las que se suprimió el riego durante tres días. En condiciones de campola enfermedad sólo hasido observadaen losestados finales de maduración del arroz, por lo que se presume que la edad de la planta es otro factor predisponente.

Con respecto a la descoloración o manchado de los granos atribuible a $\boldsymbol{H}$. oryzae $(1,8,9)$, las inoculaciones realizadas no permitieron obtener conclusiones, debiendo realizarse pruebas de patogenicidad orientadas a aclarar la participación del hongo en este problema.

Por lo expuesto, se considera que $\boldsymbol{H}$. oryzae (nomen confusum) es un hongo de bajo nivel de parasitismo, que se halla presente en cultivosdel nordeste de la Argentina, produciendo la enfermedad conocida como podredumbre castaño-rojiza de la vaina del arroz. El agente causal se ajusta al género Dichotomophthoropsis. M.B. Ellis.

\section{AGRADECIMIENTOS}

Las autoras expresan su reconocimiento al $\mathrm{Dr}$. Eduardo Piontelli L., de la Cátedra de Micología, Escuela de Medicina de la Universidad de Valparaíso, Chile, por la lectura crítica del manuscrito. Asimismo, agradecen a la Ing. Agr. María Graciela Cabrera de Alvarez la ejecución del dibujo en cámara clara incluido en este trabajo.

\section{REFERENCIAS}

1. Castaño, Z. J. (1985). Microorganismos asociados con el manchado del grano del arroz en Colombia. Arroz 34:22-25

2. Ellis, M. B. (1976). More Dematiaceous Hyphomycetes. Commonwealth Mycological Institute, Kew, Surrey, England.

3. Goos, R.D. (1987). Fungi with a twist: The Helicosporous Hyphomycetes. Mycologia 79:1-22

4. Gutierrez de Arriola, S.A. \& Mazzanti de Castañón, M. A. (1994). Mancha de la vaina por Rhizoctonia oryzae, en arrozales del nordeste de la Argentina. Fito-patología 29:41

5. Lee, F.N.; Gunnell, P.S. (1992). Obscure Fungal Diseases of the Foliage and Glumes. In: Webster, R. K; and Gunnell, P.S. eds. Compendium of Rice Diseases. American Phytopathological Society, St. Paul, Minnesota, USA.

6. Linder, D.H. (1931). The genus Helicoceras. Ann. Mo. Bot. Gard 18:1-8.
7. Mazzanti de Castañón, M.A.; Mazza de Gaiad, S.; Gutiérrez de Arriola, S.A. (1994). Reacción in vitro de Cultivares de Arroz a Sclerotium oryzae. Fitopatologia 29:172-177

8. Ou, S. H. (1985). Rice Diseases. 2nd. ed. Commonwealth Mycological Institute, Kew, Surrey, England.

9. Padwick, G. W. (1950). Manual of Rice Diseases. Commonwealth Mycological Institute, Kew, Surrey, England.

10. Richardson, M.J. (1979). An annotated list of seed borne diseases. 3th. ed., Commonwealth Mycological Institute, Kew, Surrey, England.

11. Tsukuba International Agricultural Training Centre.(1986). Diseases and insects of rice in the tropics. Satoh, Mito, Japan. 